\title{
Clinical Outcomes of a Neurocognitive Screening Program for Pediatric Sickle Cell Disease
}

\author{
Jeffrey Karst ${ }^{1}$, Meghan Miller ${ }^{1}$, Amy Heffelfinger ${ }^{1}$, Robert Newby ${ }^{1}$, and J. Scott $^{1}$ \\ ${ }^{1}$ Medical College of Wisconsin
}

July 24, 2020

\begin{abstract}
Objective: Sickle cell disease (SCD) is associated with neurocognitive and academic impairment. Routine screening is recommended to evaluate for possible concerns. This paper describes results from a neurocognitive screening battery and evaluates relationships with psychosocial and medical variables. Methods: Participants included 61 patients stratified among three age groups, including ages 6-7, ages 11-12, and ages 15-16. Patients completed a screening assessing cognitive performance, academic functioning, and attention (older subjects completed a task of executive function) and a measure assessing HRQL. Caregivers completed a clinical interview and forms evaluating HRQL, executive functioning, and attention. The impact of relevant medical variables and socio-economic status (SES) was also considered. Results: Between $20 \%$ and $33 \%$ of participants scored [?] 1 SD below age-based means on intellectual subtests, and between 31 and 48\% scored [?] 1 SD below grade-based means on academic subtests. Attention concerns were noted in $25 \%$ of patients, while $20-31 \%$ of parents reported executive function concerns. Neither disease subtype nor hemoglobin were associated with cognitive or academic functioning. There was an association between mean corpuscular volume (MCV) and both cognitive abilities and psychosocial health. Cognitive scores were correlated with SES, suggesting an impact of socioeconomic disparities on performance. Conclusions: Sickle Cell Disease negatively impacts cognitive and academic functioning both directly and indirectly due to pain, missed school, and through socioeconomic and racial disparities. These concerns may increase over time as disease processes and medication compliance often worsen. Longitudinal research is needed to further explore these trends.
\end{abstract}

\section{Introduction}

Sickle cell disease (SCD) is a genetic blood disorder affecting approximately 100,000 individuals in the United States ${ }^{1,2}$. Primary complications include acute and chronic pain, infection, and silent and overt stroke $^{3}$. SCD is also associated with neurocognitive impairment. Compared to healthy peers, children with SCD demonstrate deficits in intellectual functioning, verbal abilities, visual-motor and visual-spatial skills, attention and executive functioning including working memory, and processing speed ${ }^{4-9}$.

Neurocognitive deficits in children with SCD disease are associated with chronic anemia ${ }^{5,10,11}$, hypoxemia, and cerebrovascular ischemia ${ }^{9,12,13}$. While direct neurological insults are associated with a decline in neurocognitive performance ${ }^{14}$, even children without magnetic resonance imaging (MRI) abnormalities demonstrate lower intellectual functioning skills than healthy controls ${ }^{15}$. In another study, participants' neuropsychological performance and imaging abnormalities were not directly related, but abnormal imaging was associated with variability in cognitive performance ${ }^{16}$. Transcranial doppler (TCD) velocities were predictive of syntactical skills after controlling for anemia severity ${ }^{17}$, and there is evidence for a relationship between TCD velocity and both visual spatial and perceptual reasoning ${ }^{11}$.

In line with neurocognitive deficits, academic underachievement is common for children with $\mathrm{SCD}^{9,18,19}$. Approximately $60 \%$ of children with SCD report academic difficulties related to their disease ${ }^{20}$. Academic 
deficits in SCD are associated with overt and silent stroke $\mathrm{s}^{13,18,21}$, and school absenteeism ${ }^{22,23}$. Likely related to increased school absenteeism over the lifespan, academic problems also seem to increase over time ${ }^{24}$.

Socioeconomic status (SES) also impacts cognitive and academic functioning in children with SCD. Researchers have identified a relationship between SES and full-scale IQ, language and verbal reasoning, visual spatial and perceptual reasoning, and executive functioning ${ }^{11}$, as well as between lower SES and increased academic difficulties in children with $\mathrm{SCD}^{18,24}$. In addition, increased parenting stress germane to low SES is associated with less responsive parenting, which in turn was associated with lower cognitive functioning in children ${ }^{25}$.

Routine screening is thus recommended to assess for cognitive and academic concerns in children with $\mathrm{SCD}^{6,22}$, and our institution instituted a neurocognitive screening program in 2017. This paper describes the outcomes of these evaluations, with screening methodology and referral outcomes more explicitly discussed in a separate manuscript (in preparation). The primary aim of this study was to describe the neurocognitive and academic functioning in children with SCD and explore how functioning might vary by a function of medical variables, psychosocial factors, and age. We hypothesized that neurocognitive and academic functioning in children with SCD would be lower than ex and demonstrate a direct relationship with hemoglobin and TCD velocity, consistent with prior research. We also hypothesized that SES and psychosocial factors would have both a direct and indirect impact on neurocognitive and academic performance.

\section{Methods}

\section{Setting}

Neurocognitive screeners were scheduled for patients at four primary age points, including between 3-4 years of age, 6-7 years of age, 11-12 years of age, and 15-16 years of age (broadly reflecting periods of school transition). This paper describes results only from the latter three age points as the first age period consists of different measures. Patients most commonly completed screeners during the same date as their scheduled medical clinic visit.

Measures

\section{Psychosocial Interview}

Caregivers and patients completed a brief unstructured interview. The primary domains assessed during interviews were school performance, mood, emotional and social well-being, pain, sleep, and activity; with responses used to provide background information for reports, contextualize data from testing, and guide referrals.

\section{Demographic Form}

Parents also completed a basic demographic form. Parents were asked about school information (including absenteeism) and whether the child had an individualized education program (IEP) or 504 Plan. In addition, a list of school-related concerns including subject-based concerns (reading, writing, math), academic skill concerns (attention, memory, working speed, organization), and behavioral concerns was listed with a binary choice of "yes" or "no."

\section{Wechsler Intelligence Scale for Children - Fifth Edition (WISC-V)}

The WISC-V ${ }^{26}$ assesses intellectual functioning across five domains. For the purposes of this screening program, one test was administered from each domain, including Vocabulary (Verbal Comprehension), Block Design (Visual Spatial), Matrix Reasoning (Fluid Reasoning), Digit Span (Working Memory), and Coding (Processing Speed). Each subtest raw score produced a scaled score with a mean of 10 and anSD of 3 . An average scaled score was calculated for each participant by computing an average of the five scaled scores (reported as "mean cognitive score"). The WISC-V has established psychometric properties and has demonstrated construct invariance among African American students ${ }^{27}$.

Woodcock-Johnson Tests of Achievement - Fourth Edition (WJ-4) 
The WJ- $4{ }^{27}$ provides assessment of academic achievement across the domains of reading, written language, and mathematics. For this screening program, the Letter-Word Identification subtest was used to briefly assess reading while the Spelling subtest was used to measure verbal encoding. For the mathematics domain, the first 14 participants completed the Applied Problems subtest after which, to reduce screener duration, the Calculation subtest was substituted. Post-hoc analysis did not suggest significant differences between sores on these two tests. Depending on math subtest, WJ-4 scoring software provided either a Brief Achievement Score or Academic Skills Score; this sum score was used for each participant as a proxy for overall academic functioning (reported as "mean academic score"). All subtest and summary scores are calculated as standard scores with a mean of 100 and a $S D$ of 15 . The WJ-4 demonstrates generally appropriate psychometric properties $^{28}$.

NEPSY - Second Edition (NEPSY-2)

The Auditory Attention and Response Set subtests from the NEPSY-II ${ }^{28}$ were given to screen for concerns with attention (Auditory Attention) and executive function (Response Set). Both tasks involve focused attention on a brief auditory task, the latter task also requires the ability to shift and maintain a new set of rules. Children completing the screener who were 6 years old completed Auditory Attention only. The NEPSY-II exhibits appropriate psychometric properties ${ }^{29}$.

\section{Pediatric Quality of Life Inventory Version 4 (PedsQL 4.0 Generic Core) Parent and Child-Report}

The PedsQL 4.0 is a rating scale that assesses health-related quality of life (HRQL) in healthy children and adolescents as well as those with chronic medical conditions ${ }^{30}$. Both the caregiver and child completed the 23-item PedsQL 4.0 Generic Core questionnaire to assess reported physical, emotional, social, and school functioning. The child self-report version of the PedsQL has versions for each age range (5-7, 8-12, and 13-18). Likert-type items are reverse-scored and transformed to a 0-100 scale so that higher scores indicate greater health-related quality of life. Summary scores including total health, physical health, and psychosocial health were also calculated and established cutoff scores were used to assess scores outside of the normal range ${ }^{31}$. The PedsQL has demonstrated appropriate reliability and validity in use with this population ${ }^{32}$.

Behavior Rating Inventory of Executive Function - Second Edition (BRIEF-2)

The BRIEF-2 is a rating scale that assesses executive function and self-regulation in children and teens ages 5-18 years of age ${ }^{33}$. Caregivers completed the BRIEF-2 parent form. Within this 63 -item rating scale, behavioral regulation, emotional regulation index, and cognitive regulation were evaluated to obtain an overall Global Executive Composite. Scores on the BRIEF-2 are calculated as T-scores with a mean of 50 and an $S D$ of 10, with higher scores indicating poorer executive functioning

\section{Conners-Third Edition (Conners-3) Parent Short Form}

The Conners-3 Parent Short Form is a rating scale that assesses diagnostic symptoms of inattention and hyperactivity/impulsivity in children and teens ages 6-18 years of age ${ }^{34}$. Caregivers completed the parent short form version of the rating scale. Within this 45 -item rating scale, inattention, hyperactivity/impulsivity, learning problems, executive functioning, defiance/aggression, as well as peer relations were evaluated. Scores on the Conners- 3 are calculated as T-scores with a mean of 50 and an $S D$ of 10 , with higher scores representing greater concerns.

\section{Relevant Medical Variables}

Chart abstraction was used to obtain specific SCD genotype, overall hemoglobin, sickle hemoglobin, fetal hemoglobin, and mean corpuscular volume $(\mathrm{MCV})$, with all lab values taken $+/-45$ days of testing. In addition, for participants who had undergone a TCD, the most recent values for left and right middle cerebral artery (MCA) arteries were included as this is a region of heightened risk for stenosis and silent stroke ${ }^{35}$. Disease genotype was used as a proxy measure of disease severity, as Hemoglobin SS disease (HbSS) and Hemoglobin S beta zero thalassemia $\left(\mathrm{HbSB}^{0}\right)$ are typically characterized as more severe forms of disease while Hemoglobin SC disease (HbSC) and Hemoglobin S beta plus thalassemia $\left(\mathrm{HbSB}^{+}\right)$are considered less severe 
disease subtypes ${ }^{1}$. Overall hemoglobin, sickle hemoglobin, fetal hemoglobin, and mean corpuscular volume were included given evidence of an association between anemia and neurocognitive performance ${ }^{5,8,36}$. Mean corpuscular volume (MCV) was also included in analyses as a crude indicator of hydroxyurea compliance ${ }^{37}$.

\section{Sociodemographic Variables}

Chart abstraction was used to obtain the family address and then to calculate an estimate of SES disadvantage via the Area Deprivation Index (ADI), modified by the University of Wisconsin - Madison Neighborhood Atlas $^{38}$. The ADI provides information regarding neighborhood SES via metrics including income, education, employment, and housing quality. The ADI has been found to predict readmission risk ${ }^{39,40}$ and mortality ${ }^{41}$. For this study, national percentiles with block group levels were used with a 1-100 scale, with higher percentiles/blocks representing higher levels of disadvantage.

\section{Procedures}

The screening protocol was developed as a clinical program by the authors, which include a pediatric psychologist, two pediatric neuropsychologists, a pediatric hematologist, and a clinical psychometrist. This associated study was approved by the Institutional Review Board and appropriate informed consent and assent were obtained prior to study enrollment. Patients completed the screening program regardless of study participation. The psychologist completed the semi-structured interview with the patient's caregiver while the clinical psychometrist administered the complete battery of neurocognitive assessments to the patient.

\section{Participants}

Participants included 61 patients stratified among three age groups, with 27 patients between 6 and 7 years of age, 23 patients between 11 and 12 years of age, and 11 patients between 15 and 16 years of age. One family declined participation in the screening protocol, two patients were seen for the clinical screener but declined research participation, and two more patients were seen for the screener having but did not have a legal guardian present to consent for research participation. Sixteen patients did not show for their scheduled screener appointment and 11 cancelled without rescheduling prior to this analysis. Patients with a history of overt stroke were excluded from this analysis as they were directly referred to neuropsychology; there were five participants with a verified history of silent stroke but none with recent abnormal or conditional TCD results. There was a slight overrepresentation of females (62\%). Most patients had hemoglobin SS disease $(64 \%)$ or hemoglobin SC disease $(23 \%)$. Most patients were prescribed hydroxyurea $(\mathrm{n}=40)$; while nine were undergoing chronic transfusions due to silent stroke history $(\mathrm{n}=5)$, pain $(\mathrm{n}=3)$, and priapism $(\mathrm{n}=$ 1). Most patients attended public schools (75\%), 30\% had an IEP, and $36 \%$ had a 504 Plan. For complete demographic information, see Table 1.

\section{Data Analysis}

Test data, medical data, and demographic was entered into a database. Descriptive statistics were run for all variables of interest. Pearson correlations, t-tests, and ANOVAs were used to evaluate relationships between test scores and medical variables where appropriate. All data analysis was completed in SPSS, Version 24.0.

\section{Results}

\section{Cognitive and Academic Functioning}

Performance on WISC-V subtests was variable within and among subjects. At the individual level, mean discrepancy between participant's highest and lowest subtest scores was 4.64 scaled score points. Fifty-nine percent $(\mathrm{n}=36)$ patients had at least one subtest scaled score lower than $1 S D$ below the mean (i.e. a scaled score [?] 6), with the highest proportion of low scores on the Vocabulary subtest (33\%) followed by the Digit Span subtest $(28 \%)$. Twenty-one percent of participants demonstrated a mean cognitive score more than $1 S D$ below age-based means. Differences in mean cognitive scores among age cohorts were present but not statistically significant $(6-7$ cohort $\mathrm{M}=8.82, S D=1.70 ; 11-12$ cohort $\mathrm{M}=7.78, S D=1.65 ; 15-16$ cohort $\mathrm{M}=7.65, S D=1.91 ; F(2,58=2.45, p=.095)$. 
The mean academic score among patients on the WJ-4 was $86.03(S D=14.76)$, with $43 \%$ of patients demonstrating mean academic scores greater than $1 S D$ below the mean (SS [?] 85) compared to an expected distribution of $16 \%$ one $S D$ below the mean. Among domains, the highest proportion of standard scores greater than $1 S D$ below the mean (SS [?] 85) was on the math subtest (Calculation or Applied Problems; $48 \%$ ), while $52.5 \%(\mathrm{n}=32$ ) of participants had at least one of three subtests with a standard score $1 S D$ below the mean. Mean academic scores were significantly lower among older age cohorts, $(\mathrm{F}(1,57)=3.73, p$ $=.030)$, from an average standard score of 91.2 in the 6 - to 7-year-old group, to 83.4 in the 11- to 12-year-old group, to 78.6 (15- to 16-year-old group).

\section{Attention 83 Executive Function}

On the NEPSY-II Auditory Attention subtest, $25 \%$ of patients (total $\mathrm{n}=57$ ) performed at one $S D$ or more below the mean (i.e., scaled score [?] 6), while $18 \%$ of patients (total $\mathrm{n}=38$ ) performed in this range on the Response Set subtest. Both NEPSY-II subtests were correlated with cognitive mean scores, while only Response Set was associated with academic mean scores. For a review of WISC-V, WJ-4, and NEPSY-2 scores, see Table 2.

On the BRIEF-2, parent report suggested $20 \%$ of patients had executive functioning concerns in the mildly to clinically elevated range ( $\mathrm{T}$ score [?] 60). On the Conners-3, parent report suggested that $20 \%$ of patients had concerns with inattention, $12 \%$ had concerns related to hyperactivity, and $31 \%$ had concerns related to executive functioning. For review of mean scores, see Table 3. Parent report of executive functioning (on the BRIEF-2 and Conners-3) and attention concerns (on the Conners-3) were correlated with one another, but not associated with cognitive or academic mean scores.

\section{Cognitive/Academic/Executive Functioning and Medical Variables}

There were no statistically significant differences found between more severe subtypes (Hemoglobin SS or Hemoglobin Beta 0) and less severe types of SCD (Hemoglobin SC or Hemoglobin Beta + ) in terms of mean cognitive score, mean academic score, or parent-reported global executive functioning. Mean cognitive scores, cognitive subtest variance, cognitive subtest range, and mean academic scores were not related to overall hemoglobin, sickle hemoglobin, or fetal hemoglobin levels that were taken +/- 45 days of testing, nor were mean cognitive or academic score related to TCD right or left MCA velocities; though of note, all study participants with a TCD $(\mathrm{n}=40)$ had results in the normal range. Patients with mean academic scores $1 S D$ below the mean demonstrated marginally significant differences in left MCA velocity $(t(37)$ $=-1.82, p=.076)$. There was a significant, positive association between mean corpuscular volume (MCV) and mean cognitive score $(\mathrm{r}=.29, p=.03)$, but not between MCV and academic performance; however, patients with mean academic scores $1 \mathrm{SD}$ or below the mean had lower mean $\mathrm{MCV}(\mathrm{t}(53)=2.66, p=$ $.01)$. None of the medical variables assessed demonstrated positive associations with auditory attention scaled scores on the NEPSY-II. Similarly, neither hemoglobin nor MCV were significantly correlated with emotional, cognitive, or behavioral regulation T-scores on the BRIEF-2 or with inattention or hyperactivity T-scores on the Conners-3. Of note, there was a significant correlation found between MCV and both parent $(\mathrm{r}=.28, p=.05)$ and self-reported $(\mathrm{r}=.45, p=.001)$ psychosocial health summary, but not the physical health summary, on the PedsQL. Neither cognitive nor academic functioning were correlated with parent or child reports of pain on the PedsQL.

\section{Qualitative Parent Report}

Parent report on the demographic survey suggested academic concerns regarding math (30\%), reading (26\%), and writing skills (13\%). Parent-reported concerns regarding cognitive and learning abilities included attention (24\%), memory (24\%), organization (16\%), and working speed (15\%). Based on parent report, $36 \%$ of participants had missed 3 or more days of school in the prior 4 weeks. Follow-up analysis comparing the participants who had missed 2 or fewer days of school $(\mathrm{n}=32)$ and those who had missed 3 or more $(\mathrm{n}=18)$ did not reveal statistically significant differences on summary variables of cognitive and academic performance. However, mean academic scores were approximately seven points lower for the high absentee group (Low missed school $\mathrm{M}=89.06, S D=14.10$; Higher missed school $\mathrm{M}=81.89, S D=15.27$ ). 


\section{Health-Related Quality of Life (HRQL)}

Parent report of total HRQL was positively correlated with both mean cognitive score $(\mathrm{r}=.310, p=$ $.023)$ and mean academic score $(\mathrm{r}=.323, p=.018)$; but child reported HRQL scores did not demonstrate a significant relationship with cognitive or academic mean scores. Statistically significant differences on cognitive mean scores $(\mathrm{t}(51)=2.18, p=.035)$ and academic mean scores $(\mathrm{t}(52)=2.65, p=.011)$ were found when comparing performance between patients rated above and below the cutoffs for parent reported total HRQL (Varni et al., 2003). For review of scores, see Table 3.

\section{Sociodemographic Variables}

Participant and family socioeconomic status, as measured by the ADI, was significantly correlated with mean cognitive score $(\mathrm{r}=-.32, \mathrm{p}=.013)$ on the WISC-V but was not associated with mean academic score on the WJ-4. These results suggest that children living in more socioeconomically disadvantaged areas demonstrated overall poorer performance in cognitive but not academic functioning. Area Deprivation Index percentiles were not associated with BRIEF-2 global executive functioning T-scores, Conners-3 subscale scores for inattention or hyperactivity, or child self-report HRQL scores. However, ADI percentiles were significantly inversely correlated with parent reported total HRQL scores on the PedsQL $(\mathrm{r}=-.311, p=$ $.025)$.

\section{Conclusions}

Results of this study suggest that a high proportion of children with SCD experience neurocognitive and academic difficulties. On cognitive testing, $59 \%$ of participants had one or more subtest scores fall at least one $S D$ below age-based means; while on academic testing $52.5 \%$ of participants had at least one subtest score fall one $S D$ or more below grade-based means. A concerning trend demonstrated lower academic mean scores by older age cohorts. Correlational analysis suggests that at least a portion of this academic difficulty is associated with missed school, suggesting that the increase in SCD symptomology over time ${ }^{42}$, combined with the cumulative impact of missed school, leads to a negative cycle of academic underachievement in children with SCD. Results of testing suggested a subset of patients with attention (25\%) and executive functioning concerns (18\%) and a similar range of patients (20-31\%) with parent-reported concerns related to executive functioning. Difficulties on a subtest of attention were associated with lower cognitive scores, while difficulties on a subtest of executive functioning were associated with lower cognitive and academic scores.

In contrast to most previously published findings neither cognitive mean scores, academic mean scores, nor attention were associated with disease severity, hemoglobin, or TCD results in this sample. There was a correlation identified between mean cognitive scores and MCV, but MCV was not related to academic scores. It is possible that prior efforts in our program to identify and treat those are high medical risk of neurocognitive difficulties has served to mitigate this relationship. Cognitive mean scores were associated with SES as measured by

the ADI, but academic mean scores were not associated with SES. This finding could suggest that academic scores are more impacted by racial disparities, which are high in our region and can make it challenging for families to secure and maintain quality education, than by socioeconomic factors specifically, in line with prior research ${ }^{43,44}$.

\section{Limitations}

There are several limitations to this study. The high rate of cancellations and no-shows possibly resulted in a non-representative sample, particularly given socioeconomic barriers to attendance. The clinical nature of our program also created minor variance amongst test setting and screening measures. The lack of a control group for our sample precludes comparison to other children affected by chronic illness and/or socioeconomic disadvantage. Also, the cross-sectional nature of this study limits the extent to which we can make causal inferences or definitive conclusions about trajectories, including the poorer performance seen in older age groups. In addition, because this screening was designed for brevity, additional information (e.g., 
in-depth measures of executive functioning, verbal learning, and visual-motor integration, and/or teacher report) related to was not obtained.

\section{Future Directions}

The findings of this study suggest several important areas of future research. Most importantly, it will be essential to better understand the trajectory of neurocognitive and academic functioning over time in children with SCD and to assess for treatment and social variables that may impact this pathway. Further, it will be helpful to better understand how treatment type, adherence, and interventions might be helpful in addressing the physical and psychosocial factors affecting cognitive and academic functioning in children with SCD.

\section{Conclusion}

In summary, the screening program implemented at our institution for children with pediatric SCD identified cognitive, academic, and psychosocial concerns warranting intervention in most participants. It appears very important to implement similar programming across institutions and to better understand approaches that can be taken to mitigate the negative cognitive, academic, and social-emotional impact of SCD.

Conflict of Interest statement: We have no conflicts of interest to disclose.

Acknowledgements: We would like to thank the patients and families who participated in this study as well as the entire Sickle Cell Disease team at Children's Wisconsin for their collaboration and support. The first author would also like to express appreciation for the guidance and mentorship of Dr. Kristin Bingen and Dr. Jenny Hoag.

\section{References}

1. Brousseau DC, Panepinto JA, Nimmer M, Hoffmann RG. The number of people with sickle-cell disease in the United States: national and state estimates. Am J Hematol . 2010;85(1):77-78.

2. Kavanagh PL, Sprinz PG, Vinci SR, Bauchner H, Wang CJ. Management of Children With Sickle Cell Disease: A Comprehensive Review of the Literature. PEDIATRICS . 2011;128(6):e1552-e1574. doi:10.1542/peds.2010-3686

3. Centers for Disease Control and Prevention. Data and Statistics on Sickle Cell Disease. Published 2019. Accessed June 23, 2020. https://www.cdc.gov/ncbddd/sicklecell/index.html

4. Acquazzino MA, Miller M, Myrvik M, Newby R, Scott JP. Attention deficit hyperactivity disorder in children with sickle cell disease referred for an evaluation. J Pediatr Hematol Oncol . 2017;39(5):350-354.

5. Kral MC, Brown RT, Hynd GW. Neuropsychological aspects of pediatric sickle cell disease. Neuropsychol Rev . 2001;11(4):179-196.

6. Noll R. Neuropsychological functioning in youth with sickle cell disease: Comparison with non-chronically ill peers. J Pediatr Psychol . 2001;26(2):69-78. doi:https://doi.org/10.1093/jpepsy/26.2.69

7. Schatz J, Finke R, Roberts CW. Interactions of Biomedical and Environmental Risk Factors for Cognitive Development: A Preliminary Study of Sickle Cell Disease. 2004;25(5):8.

8. Steen RG, Fineberg-Buchner C, Hankins G, Weiss L, Prifitera A, Mulhern RK. Cognitive Deficits in Children With Sickle Cell Disease.J Child Neurol . 2005;20(2):102-107. doi:10.1177/08830738050200020301

9. Wills KE, Nelson SC, Hennessy J, et al. Transition Planning for Youth With Sickle Cell Disease: Embedding Neuropsychological Assessment Into Comprehensive Care: TABLE 1. Pediatrics . 2010;126(Supplement 3):S151-S159. doi:10.1542/peds.2010-1466J

10. Hijmans CT, Fijnvandraat K, Grootenhuis MA, et al. Neurocognitive deficits in children with sickle cell disease: a comprehensive profile.Pediatr Blood Cancer . 2011;56(5):783-788. doi:10.1002/pbc.22879 
11. Prussien KV, Siciliano RE, Ciriegio AE, et al. Correlates of Cognitive Function in Sickle Cell Disease: A Meta-Analysis. J Pediatr Psychol . 2020;45(2):145-155. doi:10.1093/jpepsy/jsz100

12. Brown RT, Davis PC, Lambert R, Hsu L, Hopkins K, Eckman J. Neurocognitive Functioning and Magnetic Resonance Imaging in Children With Sickle Cell Disease. J Pediatr Psychol . 2000;25(7):503-513. doi:10.1093/jpepsy/25.7.503

13. Schatz J, Brown RT, Pascual JM, Hsu L, DeBaun MR. Poor school and cognitive functioning with silent cerebral infarcts and sickle cell disease. Neurology . 2001;56(8):1109-1111. doi:10.1212/WNL.56.8.1109

14. Berkelhammer LD, Williamson AL, Sanford SD, et al. Neurocognitive Sequelae of Pediatric Sickle Cell Disease: A Review of the Literature.Child Neuropsychol . 2007;13(2):120-131. doi:10.1080/09297040600800956

15. Kawadler JM, Clayden JD, Clark CA, Kirkham FJ. Intelligence quotient in paediatric sickle cell disease: a systematic review and meta-analysis. Dev Med Child Neurol . 2016;58(7):672-679. doi:10.1111/dmcn.13113

16. Grueneich R. Relationship of Structural Magnetic Resonance Imaging, Magnetic Resonance Perfusion, and Other Disease Factors to Neuropsychological Outcome in Sickle Cell Disease. J Pediatr Psychol . 2004;29(2):83-92. doi:10.1093/jpepsy/jsh012

17. Sanchez CE, Schatz J, Roberts CW. Cerebral blood flow velocity and language functioning in pediatric sickle cell disease. J Int Neuropsychol Soc . 2010;16(2):326-334. doi:10.1017/S1355617709991366

18. Epping AS, Myrvik MP, Newby RF, Panepinto JA, Brandow AM, Scott JP. Academic attainment findings in children with sickle cell disease. J Sch Health . 2013;83(8):548-553.

19. Routhieaux J, Sarcone S, Stegenga K. Neurocognitive Sequelae of Sickle Cell Disease: Current Issues and Future Directions. J Pediatr Oncol Nurs . 2005;22(3):160-167. doi:10.1177/1043454205275408

20. Crosby LE, Joffe NE, Irwin MK, et al. School Performance and Disease Interference in Adolescents with Sickle Cell Disease. Phys Disabil Educ Relat Serv . 2015;34(1):14-30. doi:10.14434/pders.v34i1.13918

21. Wang W, Enos L, Gallagher D, et al. Neuropsychologic performance in school-aged children with sickle cell disease: A report from the Cooperative Study of Sickle Cell Disease. J Pediatr . 2001;139(3):391-397. doi:10.1067/mpd.2001.116935

22. Mayes S, Wolfe-Christensen C, Mullins LL, Cain JP. Psychoeducational Screening in Pediatric Sickle Cell Disease: An Evaluation of Academic and Health Concerns in the School Environment. Child Health Care . 2011;40(2):101-115. doi:10.1080/02739615.2011.566465

23. Schwartz LA, Radcliffe J, Barakat LP. Associates of school absenteeism in adolescents with sickle cell disease. Pediatr Blood Cancer . 2009;52(1):92-96. doi:10.1002/pbc.21819

24. Ezenwosu OU, Emodi IJ, Ikefuna AN, Chukwu BF, Osuorah CD. Determinants of academic performance in children with sickle cell anaemia. BMC Pediatr . 2013;13(1):189.

25. Yarboi J, Prussien KV, Bemis H, et al. Responsive Parenting Behaviors and Cognitive Function in Children With Sickle Cell Disease.J Pediatr Psychol . 2019;44(10):1234-1243. doi:10.1093/jpepsy/jsz065

26. Wechsler D, Kaplan E. WISC-V Integrated Technical and Interpretive Manual . Pearson; 2015.

27. Schrank FA, Mather N, McGrew KS. Woodcock-Johnson IV Tests of Achievement . Riverside; 2014.

28. Korkman M, Kirk U, Kemp S. NEPSY II: Clinical and Interpretive Manual. Harcourt Assessment, PsychCorp; 2014.

29. Brooks BL, Sherman EM, Strauss E. Child Neuropsychol . 2009;16(1):80-101. 
30. Varni JW, Seid M, Kurtin PS. PedsQL ${ }^{\mathrm{TM}}$ 4.0: Reliability and Validity of the Pediatric Quality of Life Inventory $^{\mathrm{TM}}$ Version 4.0 Generic Core Scales in Healthy and Patient Populations: Med Care . 2001;39(8):800812. doi:10.1097/00005650-200108000-00006

31. Varni JW, Burwinkle TM, Seid M, Skarr D. The PedsQL* 4.0 as a Pediatric Population Health Measure: Feasibility, Reliability, and Validity. Ambul Pediatr . 2003;3:329-341.

32. Panepinto JA, Bonner M. Health-related quality of life in sickle cell disease: Past, present, and future. Pediatr Blood Cancer . 2012;59(2):377-385. doi:10.1002/pbc.24176

33. Gioia GA, Isquith PK, Guy SC, Kenworthy L. BRIEF-2: Behavior Rating Inventory of Executive Function: Professional Manual . Psychological Assessment Resources; 2015.

34. Conners CK. Conners Comprehensive Behavior Rating Scales: Manual . Multi-Health Systems; 2008.

35. Adams RJ. TCD in sickle cell disease: an important and useful test.Pediatr Radiol . 2005;35(3):229-234. doi:10.1007/s00247-005-1409-7

36. Hijmans CT, Grootenhuis MA, Oosterlaan J, Heijboer H, Peters M, Fijnvandraat K. Neurocognitive deficits in children with sickle cell disease are associated with the severity of anemia. Pediatr Blood Cancer . 2011;57(2):297-302. doi:10.1002/pbc.22892

37. Brandow AM, Panepinto JA. Monitoring toxicity, impact, and adherence of hydroxyurea in children with sickle cell disease. Am J Hematol . 2011;86(9):804-806. doi:10.1002/ajh.22101

38. University of Wisconsin. Neighborhood Atlas - Home. Neighborhood Atlas. Published 2020. Accessed June 24, 2020. https://www.neighborhoodatlas.medicine.wisc.edu/

39. Hu J, Kind AJH, Nerenz D. Area Deprivation Index Predicts Readmission Risk at an Urban Teaching Hospital. Am J Med Qual . 2018;33(5):493-501. doi:10.1177/1062860617753063

40. Kind AJH, Jencks S, Brock J, et al. Neighborhood Socioeconomic Disadvantage and 30-Day Rehospitalization: A Retrospective Cohort Study.Ann Intern Med . 2014;161(11):765. doi:10.7326/M13-2946

41. Singh GK. Area Deprivation and Widening Inequalities in US Mortality, 1969-1998. Am J Public Health . 2003;93(7):1137-1143. doi:10.2105/AJPH.93.7.1137

42. Brandow AM, Zappia KJ, Stucky CL. Sickle cell disease: a natural model of acute and chronic pain. PAIN . 2017;158:S79-S84. doi:10.1097/j.pain.0000000000000824

43. Dotson VM, Kitner-Triolo MH, Evans MK, Zonderman AB. Effects of race and socioeconomic status on the relative influence of education and literacy on cognitive functioning. J Int Neuropsychol Soc . 2009;15(4):580-589. doi:10.1017/S1355617709090821

44. Smith EP, Atkins J, Connell CM. Family, School, and Community Factors and Relationships to Racial-Ethnic Attitudes and Academic Achievement. Am J Community Psychol . 2003;32(1-2):159-173. doi:10.1023/A:1025663311100

\section{Hosted file}

Table1. docx available at https: //authorea. com/users/345778/articles/471903-clinical-outcomesof-a-neurocognitive-screening-program-for-pediatric-sickle-cell-disease

\section{Hosted file}

Table2 . docx available at https://authorea. com/users/345778/articles/471903-clinical-outcomesof-a-neurocognitive-screening-program-for-pediatric-sickle-cell-disease

\section{Hosted file}


Table3. docx available at https://authorea. com/users/345778/articles/471903-clinical-outcomesof-a-neurocognitive-screening-program-for-pediatric-sickle-cell-disease 